\title{
Ethiopia: who to educate, how to educate and what to teach
}

\author{
Kassa Darge • Yocabel Gorfu • Diego Jaramillo
}

Received: 19 November 2013 / Accepted: 14 February 2014

(C) Springer-Verlag Berlin Heidelberg 2014

Four major factors necessitate the support for and advancement of pediatric imaging in Ethiopia. First, the demographics are loud and clear: $44 \%$ of the population is younger than 14 years and $60 \%$ is younger than 20 years. At the largest general referral hospital of the country, Tikur Anbessa Hospital in Addis Ababa, 47\% of the patient population is children. Second, higher education in Ethiopia has witnessed historical undertakings through the establishment of more than 20 universities that have high need of well-trained professionals. Third, various medical specialties have already started or are in the process of establishing subspecialties like pediatric surgery, pediatric oncology and neonatologyspecialties that necessitate dedicated pediatric imaging support. However, there is not a single pediatric radiologist in the country, which has an estimated population of 93 million. Fourth, there is a significant discrepancy with regard to the availability of radiologic equipment between the public and private sectors. The Faculty of Medicine of the Addis Ababa University, in Tikur Anbessa Hospital, which is a public entity, runs the only radiology residency program in the country. Currently, there is no MRI scanner at this facility. Thus graduates face major challenges when they go out to work in better-equipped public or private facilities.

The Children's Hospital of Philadelphia (CHOP) Pediatric Radiology Outreach Program in Ethiopia has made the commitment to lead the efforts to support pediatric radiology education in Ethiopia. Building on the training of local

K. Darge $(\bowtie) \cdot$ D. Jaramillo

Department of Radiology, The Children's Hospital of Philadelphia,

University of Pennsylvania, 34th Street and Civic Center

Boulevard, Philadelphia, PA 19104, USA

e-mail: darge@email.chop.edu

Y. Gorfu

Department of Radiology, School of Medicine,

Addis Ababa University,

Addis Ababa, Ethiopia radiology academic faculty members through a pediatric radiology fellowship is likely to have a sustainable positive outcome. Thus efforts to implement pediatric radiology education need to consider residents, faculty members and practicing radiologists. The educational support is multifaceted and includes regular pediatric radiology visiting professorships, tele-radiology for teaching, provision of teaching cases and recorded pediatric radiology lectures, organization of an observership at our institution (supported through the Soroosh Mahboubi International Fund for Higher Education) and research mentorship.

We conduct an annual pediatric radiology continuing education course in collaboration with the Radiological Society of Ethiopia to develop pediatric imaging in the country by focusing on practicing radiologists. We emphasize how to optimize and broaden the spectrum of ultrasound studies in children. There is scarcity of CT and MRI scanners in the teaching hospitals. In the private sector these modalities are more prevalent. We specifically address pediatric $\mathrm{CT}$ and MRI techniques, indications and interpretations to help close the existing knowledge gap. In conclusion, the vision of the CHOP international outreach program is that there will be increasing and appropriate emphasis of pediatric imaging in the radiology residency, supported by the first batch of graduating local pediatric radiologists.

\section{Conflicts of interest None}

